\title{
Feasibility study on real-time $\gamma$-ray spectrum / dose measurement system
}

\author{
Mina Kobayashi ${ }^{1, *}$, Fuminobu Sato $^{1}$, Sachie Kusaka ${ }^{1}$ and Isao Murata $^{1}$ \\ ${ }^{1}$ Division of Sustainable Energy and Environmental Engineering, Graduate School of Engineering, Osaka University, Yamada-oka 2-1, \\ Suita, Osaka, 565-0871 Japan
}

\begin{abstract}
Recently, medical applications of radiation have been widely spread. However, exposure of medical staffs is sometimes not focused on because treatment of patients is the first priority. It is thus important to decrease exposure for them as much as possible. The purpose of this study is to develop a system which can measure energy spectrum and dose of gamma-rays at the same time in real time in medical application spots. As a result, the medical staff could be guided to aware the risk of radiation and finally the exposure dose to them could be suppressed substantially. We first decided to use a CsI(Tl) scintillator as the gamma-ray detection device. A Multi-Pixel Photon Counter (MPPC) was attached to the scintillator to detect gamma-ray signals. Pulse height spectra were measured with several standard gammaray sources. The detection efficiency and energy resolution were deduced from the measured results and the detection efficiency was compared with the calculation result by MCNP5. After evaluating the response function, the energy spectrum was derived with the spectrum type Bayesian estimation and the sequential Bayesian estimation procedure. From the result, it was confirmed that the sequential Bayesian estimation could be applied to real time measurement of gamma-ray energy spectrum and dose.
\end{abstract}

\section{Introduction}

In recent years, radiations have been used very often in medical field, i.e., radiation therapy, production of radioactive drugs and so on. There are risks of radiation exposure to medical staffs in the field by secondary produced gamma-rays. However, the exposure dose of medical staffs is not so seriously focused on so far, because treatment of patients is the first priority in hospitals. The medical staffs notice their radiation exposure, however, work for patients devotedly. The purpose of this study is to develop a spectrometer (monitor) which can show gamma-ray energy spectrum and the dose simultaneously in real time to make them understand the risk of radiation appropriately and to finally decrease the exposure dose of the medical staffs. The staffs will be able to engage in medical treatments with radiations, recognizing their exposure dose and energy information (gamma-ray energy spectrum) at the same time for their own safety. The monitor thus has another advantage as in the following: In the past study, there were monitors which can indicate the pulse height spectrum and/or dose [1]. However, there were no monitors which can show the gamma-ray energy spectrum and dose at the same time. The monitor which we seek estimate the energy spectrum of gamma-rays, with which the dose can be evaluated accurately.

In this paper, the result of basic performance evaluation of a prototype gamma-ray spectrum and dose measuring system and conversion procedure from the pulse height spectrum to energy spectrum are described.

\section{Measuring system}

\subsection{Design criteria of the system}

The essential conditions for designing the spectrometer are as follows.

(1) The spectrometer is small and light for practical use by medical staffs.

(2) The elemental measuring device has high detection efficiency and good energy resolution so as to reproduce even energy spectrum of gamma-rays.

(3) In regard to the dynamic range, the upper energy of gamma-rays was set to $3 \mathrm{MeV}$, because radioactive nuclides, which are produced in the operation of accelerators for particle radiation therapy or making radioisotopes for medicine, can emit gamma-rays having an upper energy of around $3 \mathrm{MeV}$.

To meet these conditions, scintillators are clearly preferable. Candidate scintillators must have a similar performance to $\mathrm{NaI}(\mathrm{Tl})$ because $\mathrm{NaI}(\mathrm{Tl})$ is regarded as a convenient and most popular detector for measuring gamma-rays among various scintillators. But $\mathrm{NaI}(\mathrm{Tl})$ has deliquescency, hence the measurement system tends to be large and heavy, because a metal case is required to keep the $\mathrm{NaI}(\mathrm{Tl})$ crystal. Therefore, instead of $\mathrm{NaI}(\mathrm{Tl})$, $\mathrm{CsI}(\mathrm{Tl})$ is used in this study, because $\mathrm{CsI}(\mathrm{Tl})$ has almost the same performance as $\mathrm{NaI}(\mathrm{Tl})$. Moreover $\mathrm{CsI}(\mathrm{Tl})$ has

\footnotetext{
* Corresponding author: mkobayashi@gr.see.eng.osaka-u.ac.jp
} 
no deliquescency. Then, we used a Multi-Pixel Photon Counter (MPPC) as a photon counting device, because the MPPC is really smaller and lighter than a photomultiplier tube (PMT).

In this study, as a prototype of the present measuring system of gamma-rays, we chose a CsI(Tl) crystal of $3 \mathrm{x}$ $3 \times 3 \mathrm{~cm}^{3}$ and measured the basic performance of the CsI(Tl) attached to the MPPC.

\subsection{Derivation of energy spectrum by Bayesian estimation}

The present measuring system utilizes a $\mathrm{CsI}(\mathrm{Tl})$ detector to measure the pulse height spectrum of gamma-rays. After the pulse height spectrum is obtained, it is converted into the energy spectrum and furthermore from the energy spectrum the dose rate is estimated with the flux-dose conversion factor. The point in this study is the energy spectrum and dose can be evaluated at the same time by conducting conversion from the pulse height spectrum to the energy spectrum in real time. This is the goal of the present study.

For this purpose, we employ the Bayesian estimation process to convert from the measured pulse height spectrum to the energy spectrum continuously in real time. In our laboratory, the spectrum type Bayesian estimation has been used for years to estimate various energy spectra, in which as the first step the pulse height spectrum is measured and thereafter it is converted into the energy spectrum as the post-process. In this study, this flow is continuously conducted for each count during measurement by the sequential Bayesian estimation [2], the detail of which is described in the following.

\subsubsection{Bayesian estimation}

The Bayes theorem is known as a conditional probability in mathematics. There are two events $A$ and $B$. The probability of $B$ is $P(B)$ and the conditional probability of $A$ for given $B$ is $P(A \mid B)$. When $P(B)$ is not zero, $P(A \mid B)$ is written by following equation.

$$
P(A \mid B)=\frac{P(B \cap A)}{P(B)}
$$

From Eq. (1), the next equation can be derived by considering $P(B \cap A)=P(A \cap B)=P(B \mid A) \cdot P(A)$.

$$
\mathrm{P}(A \mid B)=\frac{P(B \mid A) \cdot P(A)}{P(B)}
$$

In Eq. (2), when $A_{j}(j=1 \sim n)$ instead of $A$ is a mutually exclusive event and they include the whole events in the sample space $\mathrm{S}$, an arbitrary event $B$ is written by Eq. (3).

$$
\begin{aligned}
P(B)- & P\left(A_{1}\right) \cdot P\left(B \mid A_{1}\right) \\
& +P\left(A_{2}\right) \cdot P\left(B \mid A_{2}\right) \\
& +\cdots+P\left(A_{n}\right) \cdot P\left(B \mid A_{n}\right) \\
= & \sum_{j=1}^{n} P\left(A_{j}\right) \cdot P\left(B \mid A_{j}\right)
\end{aligned}
$$

From the above, a conditional probability of $A_{j}$ for given $B$ is written by Eq. (4).

$$
P\left(\Lambda_{j} \mid B\right)=\frac{P\left(B \mid A_{j}\right) \cdot P\left(A_{j}\right)}{\sum_{i=1}^{n} P\left(B \mid A_{j}\right) \cdot P\left(A_{j}\right)}
$$

This is the basic expression of the Bayesian estimation. In Eq. (4), $P\left(A_{j}\right)$ and $P\left(A_{j} \mid B\right)$ are called prior probability and posterior probability, respectively. In the general way of Bayesian estimation, first of all, a prior probability $P\left(A_{j}\right)$ is assigned an arbitrary probability value, and then $P\left(B \mid A_{j}\right)$ evaluated beforehand is substituted in Eq. (4). Finally a posterior probability $P\left(A_{j} \mid B\right)$ is obtained. By repeating this process, we can obtain more reliable probability.

In this study, by applying this essential process one after another at a time of count, we can estimate the energy spectrum in real time. Eq. (5) shows the relation of the response function, pulse height spectrum and energy spectrum in radiation measurement.

$$
\left(\begin{array}{c}
y_{1} \\
\vdots \\
y_{i} \\
\vdots \\
y_{n}
\end{array}\right)=\left(\begin{array}{cccccc} 
& & & & \\
R_{11} & R_{12} & \cdots & R_{1 j} & \cdots & R_{1 n} \\
\vdots & & \vdots & & \vdots \\
R_{i 1} & & & R_{i j} & & R_{i n} \\
\vdots & & & \vdots & & \vdots \\
R_{n 1} & \cdots & & R_{n j} & \cdots & R_{n m}
\end{array}\right) \cdot\left(\begin{array}{c}
\varphi_{1} \\
\vdots \\
\varphi_{j} \\
\vdots \\
\varphi_{n}
\end{array}\right)
$$

In Eq. (5), $y_{i}$ is the pulse height spectrum called posterior probability in the Bayesian estimation. It means, when a spectrometer detects a pulse height signal at channel $i$, the signal would come from an event originated from energy $E_{j}$ in the energy spectrum $\varphi$, which is called prior probability. $R_{i j}$ means a likelihood, which is defined as the response function of the detector for each energy, $E_{j}$, in radiation measurements. We can derive the probability of the energy spectrum at $j$ from a measured pulse height signal at $i$ with the estimated response function of the detector, $R_{i j}$, in the following equation rewritten from Eq. (4).

$$
P(j \mid i)=\frac{R_{i j} \varphi_{j}}{\sum_{j=1}^{n} R_{i j} \varphi_{j}}
$$

The numerator of the right side means the conditional relative probability of a detected signal at $i$ to be assigned to $\varphi_{j}$ as understood from Eq. (5). 


\subsubsection{Spectrum type Bayesian estimation}

A pulse height spectrum is measured in advance in the spectrum type Bayesian estimation. As in Eq. (5), it means after the measurement $y_{i}$ has a certain count. With Eq. (6), it can be expected that the posterior probability would be estimated by Eq. (7).

$$
P(j \mid i)=y_{i} \frac{R_{i j} \varphi_{j}}{\sum_{j=1}^{n} R_{i j} \varphi_{j}}
$$

By summing up $y_{i}$ for pulse height $i$, the following Eq. (8) can be obtained.

$$
P(j \mid i)=\sum_{i=1} y_{i} \frac{R_{i j} \varphi_{j}}{\sum_{j=1}^{n} R_{i j} \varphi_{j}}
$$

This is known to be an extended interpretation of the Bayesian estimation. By using the posterior probability as the prior probability, estimation can be carried out repeatedly. After an appropriate number of iterations, the converged estimation would be obtained. The spectrum type Bayesian estimation is usually applied to radiation measurements. However, in the present study, estimation should be carried out continuously during the measurement. For that purpose, the sequential Bayesian estimation was adopted instead as detailed in the next section.

\subsubsection{Sequential Bayesian estimation}

The first step of the sequential Bayesian estimation is to set an arbitrary initial spectrum to $\varphi_{j}$. We then calculate the porior probability of $\varphi$ at energy $j$ from a measured signal $y$ at channel $i$ with the response function $R_{i j}$ using Eq. (6). As a result, the $\varphi_{j}$ is adjusted by adding one contribution of the detected signal at channel $i$. By repeating this process for every count, we could obtain the converged energy spectrum, $\varphi_{j}$. However, in reality this process is known to be not properly working as detailed in Ref. 2 by Iwasaki. Then as pointed out by Iwasaki, we introduce a factor $\alpha$ which adjusts the rate of correction to the prior probability. It can control how strongly the prior can affect compared to the next posterior probability [2]. The estimation is carried out by Eq. (9).

$$
\varphi_{j}=(1-\alpha) \varphi_{j}+\alpha \cdot \frac{R_{i j} \varphi_{j}}{\sum_{j=1}^{n} R_{i j} \varphi_{j}}
$$

There is no precedent which showed the energy spectrum could be estimated accurately by the sequential Bayesian estimation. Generally, it is known that the convergence of the sequential Bayesian estimation is very slow. However we think even if the spectrum estimation is not completely converged, we can estimate the dose, because the dose is theoretically an integral quantity of the energy spectrum and it has to be converged rather faster.

\section{Experimental methods}

We made up of a prototype measuring device with a $\mathrm{CsI}(\mathrm{Tl})$ crystal $(3 \mathrm{~cm} \times 3 \mathrm{~cm} \times 3 \mathrm{~cm})$ and MPPC (C11206-4689) as shown in Fig. 1.

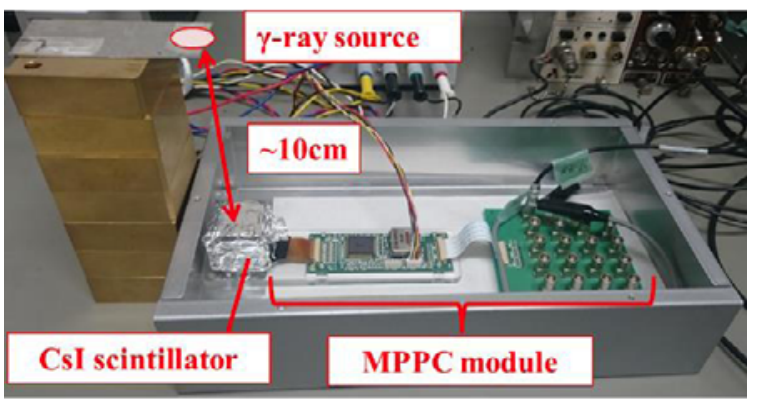

Fig. 1. Prototype system

The pulse height spectra were measured with several standard gamma-ray sources $\left({ }^{22} \mathrm{Na},{ }^{60} \mathrm{Co},{ }^{133} \mathrm{Ba},{ }^{137} \mathrm{Cs}\right.$ and $\left.{ }^{241} \mathrm{Am}\right)$ to check the detection efficiency and energy resolution. The details of the standard gamma-ray sources are summarized in Table 1.

Table 1. Detail of the standard gamma-ray sources

\begin{tabular}{cccc}
\hline Nuclide & Energy $(\mathrm{MeV})$ & Half-life & Radioactivity $(\mathrm{Bq})$ \\
\hline${ }^{22} \mathrm{Na}$ & 0.551 & $2.601 \mathrm{y}$ & $3.57 \mathrm{E}+05$ \\
& 1.274 & & \\
${ }^{60} \mathrm{Co}$ & 1.173 & $5.271 \mathrm{y}$ & $6.84 \mathrm{E}+05$ \\
& 1.332 & & \\
${ }^{133} \mathrm{Ba}$ & 0.356 & $10.52 \mathrm{y}$ & $6.84 \mathrm{E}+05$ \\
${ }^{137} \mathrm{Cs}$ & 0.662 & $30.07 \mathrm{y}$ & $2.50 \mathrm{E}+05$ \\
${ }^{241} \mathrm{Am}$ & 0.059 & $432.2 \mathrm{y}$ & $3.77 \mathrm{E}+05$ \\
\hline
\end{tabular}

The CsI(Tl) crystal was attached to a MPPC and was covered with Teflon tape and aluminum foil to shield light. They were placed in an aluminum box together with a power control module. A standard gamma-ray source was positioned at $10 \mathrm{~cm}$ from the $\mathrm{CsI}(\mathrm{Tl})$ scintillator surface. Other equipment like a power supply, multi-channel analyzer (MCA) and so on are outside the box. Fig. 2 shows the block diagram of the electronic circuit of the prototype detector. Pulse height spectra were measured by the MCA controlled by a laptop computer.

The measurements were completed when the net area count of the main peak exceeds 10000 counts. From the measured pulse height spectrum, detection efficiency and energy resolution were determined. The measured detection efficiencies were compared with simulations by MCNP5 (Monte Carlo N-Particle Transport Code) [3] to verify the validity of the code and the performance to apply to simulations of other size crystals. 


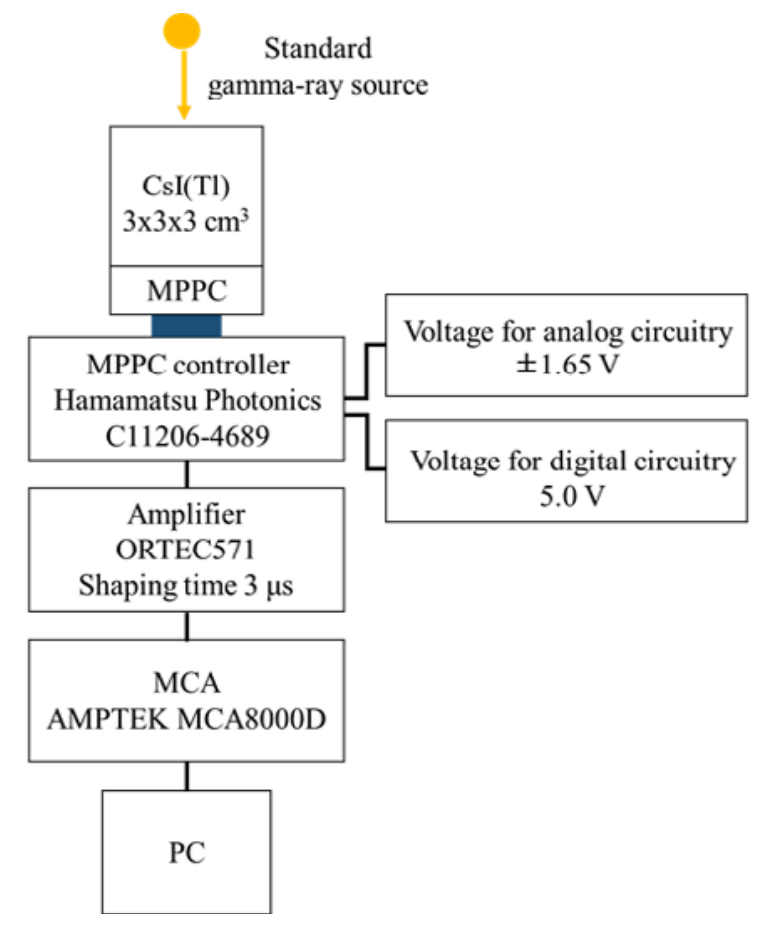

Fig. 2. Block diagram of the electronic circuit of the prototype detector.

\section{Result and discussion}

\subsection{Basic performance of CsI(TI)}

We measured pulse height spectra for ${ }^{22} \mathrm{Na},{ }^{60} \mathrm{Co},{ }^{133} \mathrm{Ba}$, ${ }^{137} \mathrm{Cs}$ and ${ }^{241} \mathrm{Am}$, and compared the results with $\mathrm{NaI}(\mathrm{Tl})$. The comparison result is described in Figs. 3 and 4. These two figures indicate the performance of the CsI(Tl) scintillator, i.e., the detection efficiency and energy resolution, was better than $\mathrm{NaI}(\mathrm{Tl})$, which is a normally utilized scintillator for gamma-ray dose measurement.

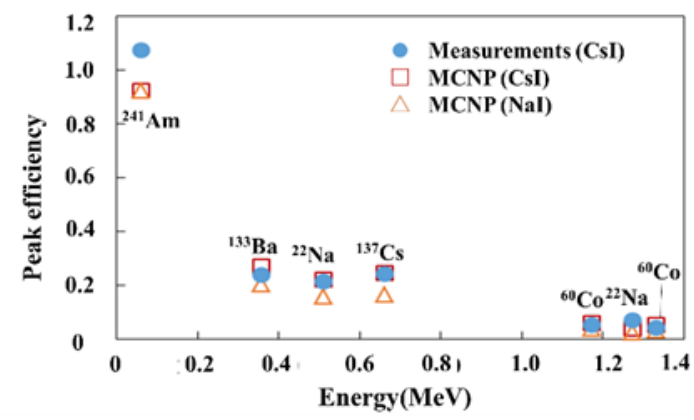

Fig. 3. Measured detection efficiency of CsI compared with the simulation result of NaI and CsI by MCNP5. The agreement is excellent except ${ }^{241} \mathrm{Am}$ between measurement and calculation for CsI. Also the performance is better than $\mathrm{NaI}$ having the same size.

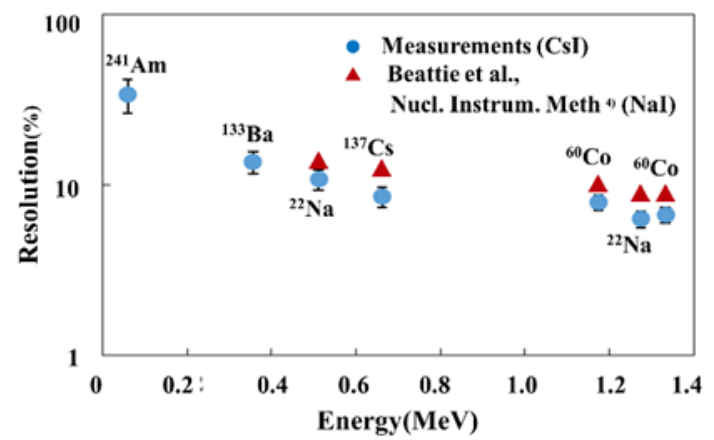

Fig. 4. Measured energy resolution of CsI compared with calculated values of $\mathrm{NaI}$ by Beattie et al. ${ }^{4)}$. The energy resolution of CsI is found to be better than $\mathrm{NaI}$.

Fig. 5 also shows comparison of the measured pulse height spectrum for ${ }^{22} \mathrm{Na}$ with the calculation result by MCNP5, in which two strong photo peaks of $0.511 \mathrm{MeV}$ and $1.27 \mathrm{MeV}$ are clearly observed. From the figure, an excellent agreement was obtained. Also, in the measured spectrum, a clear backscattering peak is observed. This should be removed in the real detector response function. In the present case, the backscattering peak was correctly reproduced by appropriately considering surrounding materials of the scintillator like a desk and other massive items. It means it would be possible to evaluate the detector response function properly by considering the precise structure of the present detector including the casing and inside. From the present comparison, the validity of the calculation code, MCNP5, was finally confirmed.



Fig. 5. Measured pulse height spectrum for ${ }^{22} \mathrm{Na}$ compared with MCNP5 calculation.

\subsection{Evaluation of response function}

To carry out the Bayesian estimation, the response function of the prototype $\mathrm{CsI}(\mathrm{Tl})$ spectrometer was evaluated. Fig. 6 shows examples of pulse height spectra for monoenergetic gamma-rays up to $3 \mathrm{MeV}$. Pulse height spectra evaluated for every $10 \mathrm{keV}$ from $100 \mathrm{keV}$ to $3 \mathrm{MeV}$ are compiled to make the response function of the present prototype spectrometer. In Fig. 6, we can find that a backscatter peak appears for each energy, and single or double escape peak appear for energies larger than around $2 \mathrm{MeV}$. Moreover especially at $3 \mathrm{MeV}$, the double escape peak is larger than the photoelectric peak. 


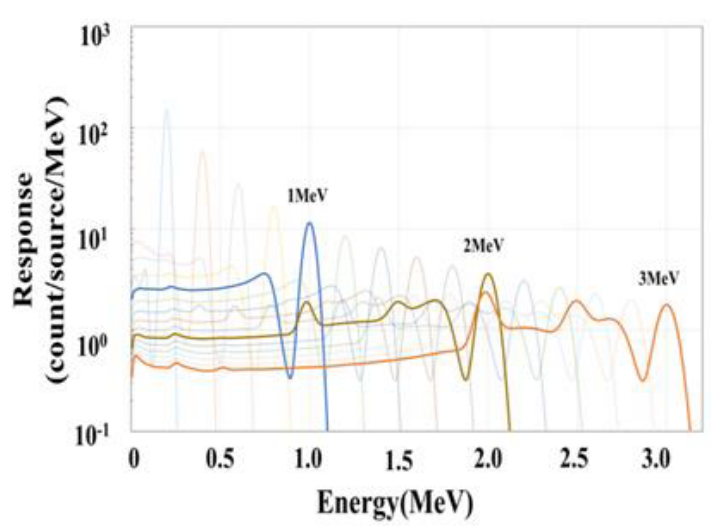

Fig. 6. The response function of a $3 \times 3 \times 3 \mathrm{~cm}^{3} \mathrm{CsI}$ scintillator to monoenergetic gamma-rays up to $3 \mathrm{MeV}$.

From the above result, the energy spectrum is clearly different from the pulse height spectrum, and cannot be estimated directly from the pulse height spectrum. Therefore the unfolding process is needed to derive the energy spectrum.

\subsection{Unfolding by Bayesian estimation}

The measured pulse height spectra were unfolded by the Bayesian estimation process to estimate the gamma-ray energy spectra with the evaluated response function described in Sec. 4.2. In this study, we carried out comparison between the result of the spectrum type Bayesian estimation and the sequential Bayesian estimation to test and confirm the performance of the latter estimation method, because the spectrum type Bayesian estimation is commonly used in radiation measurements.

Fig. 7 shows the unfolding result of the pulse height spectrum for ${ }^{22} \mathrm{Na}$ shown in Fig. 5 with the spectrum type Bayesian estimation.



Fig. 7. The energy spectrum for ${ }^{22} \mathrm{Na}$ calculated by the spectrum type Bayesian estimation.

In Fig. 7 clear two peaks of $0.511 \mathrm{MeV}$ and $1.27 \mathrm{MeV}$ are observed, which correspond to emitted two gammarays from ${ }^{22} \mathrm{Na}$. There are no any other significant peaks seen except the two peaks. Moreover the ratio of the number of emitted gamma-rays is estimated to be 0.511 $\mathrm{MeV}: 1.27 \mathrm{MeV}=1.73: 1$, which was calculated by making the ratio of the number of the peak net counts for the two peaks. The measured ratio is in good agreement with the literature value of $0.511 \mathrm{MeV}: 1.27 \mathrm{MeV}=$ $1.8: 1$.

In the sequential Bayesian estimation, at first we need to determine $\alpha$ value in Eq. (9) so as to give the most accurate result. At the same time, the minimum required number of counts should be evaluated for real applications. They were finally determined by considering the following points.

(i). Deviation between measured spectra $\left(M_{i}\right)$ and folding spectra $\left(y_{i}\right)$ of unfolding result with Eq. (5)

(ii). Reproducibility of the emission rate of gamma-rays for spectra after unfolding

In regard to point (i), the deviation for $\alpha$ value and various number of counts for ${ }^{133} \mathrm{Ba}$ is shown in Fig. 8 as an example. Here, the deviation is calculated as $1 / N \sqrt{\sum_{i}\left(M_{i}-y_{i}\right)^{2}} . N$ means the total number of counts of gamma-rays in the experiment. As Fig. 8 shows, the deviation becomes less than $1 \%$ in 50000 counts, moreover, the deviation becomes large for $\alpha>0.1$. A similar tendency was seen for other nuclides as well.

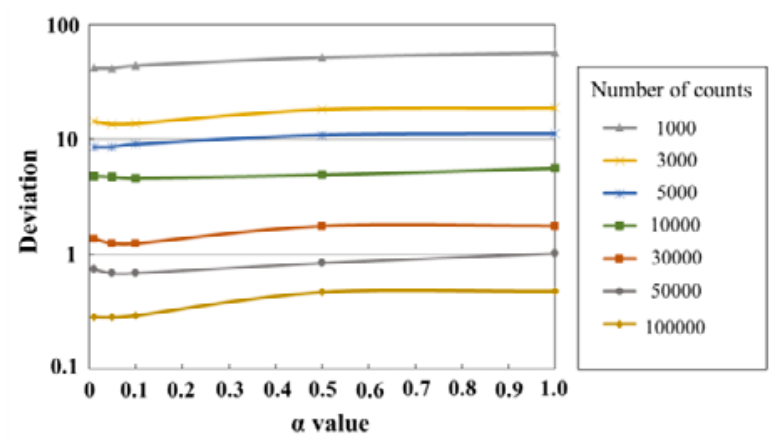

Fig. 8. Deviation for ${ }^{133} \mathrm{Ba}$ for various numbers of counts as a function of $\alpha$.

Therefore we examined point (ii) to determine the best $\alpha$ value for $\alpha \leq 0.1$. Table 2 shows $\mathrm{C} / \mathrm{E}$ indicating the reproducibility of the emission rate of gamma-rays. "C" is the evaluated number of emitted gamma-rays in the literature. "E" is the number of emitted gamma-rays summed up in each peak of the unfolded spectrum when the iteration number is 1000 in the case of the spectrum type Bayesian estimation and when the number of measured signals is $10000,50000,100000$ with the sequential Bayesian estimation.

From Table 2, the precision of the sequential Bayesian estimation is confirmed to be the same level as the spectrum type Bayesian estimation. By considering the results for the three $\alpha$ values of $0.01,0.05$ and 0.1 , the best value is 0.05 over 50000 counts. On the other hand, if examining the variance of $\mathrm{C} / \mathrm{E}$, the variance is converged for the number of counts over 50000 as shown in the bottom of Table 2, where the variance is calculated as $1 / N^{\prime} \sum(C / E-1)^{2} . N^{\prime}$ means the number of data of $\mathrm{C} / \mathrm{E}$. In conclusion, in the present detector setup, 50000 counts are consequently thought to be enough for real applications for $\alpha=0.05$. 
Table 2. Comparison of the number of emitted gamma-rays $(\mathrm{C} / \mathrm{E})$ estimated by both Bayesian methods at 1000 times iterations or 10000,50000 and 100000 counts and variance of $\mathrm{C} / \mathrm{E}$.

\begin{tabular}{|c|c|c|c|c|c|c|c|c|c|c|c|}
\hline \multirow[t]{2}{*}{ Nuclide } & \multirow{2}{*}{$\begin{array}{l}\text { Energy } \\
(\mathrm{MeV})\end{array}$} & \multirow{2}{*}{$\begin{array}{l}\text { The spectrum type } \\
\text { Bayesian estimation } \\
\text { (1000 times iterations) }\end{array}$} & \multicolumn{3}{|c|}{$\begin{array}{l}\text { The sequential Bayesian estimation } \\
\qquad(10000 \text { counts })\end{array}$} & \multicolumn{3}{|c|}{$\begin{array}{l}\text { The sequential Bayesian estimation } \\
\qquad(50000 \text { counts })\end{array}$} & \multicolumn{3}{|c|}{$\begin{array}{l}\text { The sequential Bayesian estimation } \\
\text { (100000 counts) }\end{array}$} \\
\hline & & & $\alpha=0.01$ & $\alpha=0.05$ & $\alpha=0.1$ & $\alpha=0.01$ & $\alpha=0.05$ & $\alpha=0.1$ & $\alpha=0.01$ & $\alpha=0.05$ & $\alpha=0.1$ \\
\hline \multirow[t]{2}{*}{${ }^{22} \mathrm{Na}$} & 0.511 & 1.41 & 1.25 & 1.09 & 0.98 & 1.28 & 1.16 & 1.07 & 1.51 & 1.16 & 1.10 \\
\hline & 1.274 & 1.36 & 1.55 & 1.61 & 1.74 & 1.46 & 1.28 & 1.27 & 1.10 & 1.06 & 1.10 \\
\hline \multirow[t]{2}{*}{${ }^{60} \mathrm{Co}$} & 1.173 & 1.31 & 1.38 & 1.29 & 1.23 & 1.20 & 1.38 & 1.54 & 1.19 & 1.03 & 0.92 \\
\hline & 1.332 & 1.28 & 1.32 & 1.36 & 1.25 & 1.40 & 1.05 & 0.87 & 1.27 & 1.15 & 1.20 \\
\hline${ }^{133} \mathrm{Ba}$ & 0.356 & 0.72 & 0.80 & 0.77 & 0.60 & 0.76 & 0.68 & 0.48 & 0.65 & 0.54 & 0.49 \\
\hline${ }^{137} \mathrm{Cs}$ & 0.662 & 0.81 & 0.82 & 0.75 & 0.73 & 0.80 & 0.71 & 0.68 & 0.77 & 0.69 & 0.68 \\
\hline \multicolumn{2}{|c|}{ Variance of $\mathrm{C} / \mathrm{E}$} & 0.10 & 0.11 & 0.12 & 0.15 & 0.10 & 0.07 & 0.13 & 0.09 & 0.06 & 0.07 \\
\hline
\end{tabular}

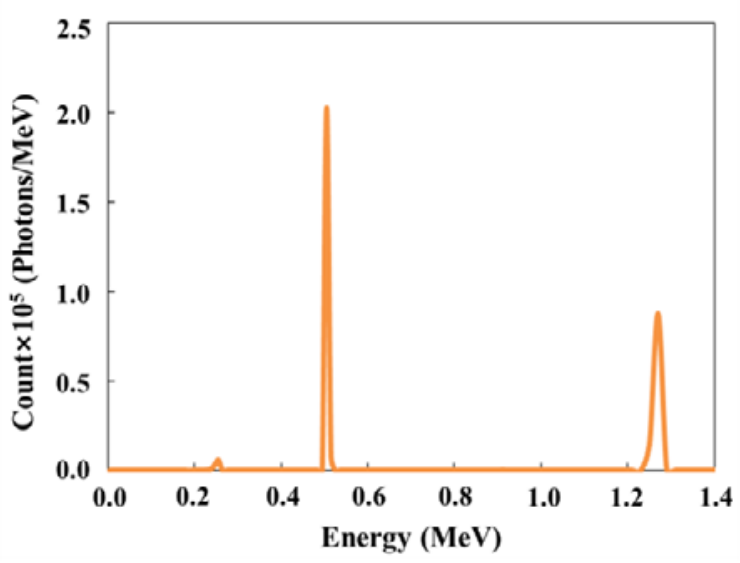

Fig. 9. The energy spectrum for ${ }^{22} \mathrm{Na}$ obtained by unfolding by the sequential Bayesian estimation when the number of measured signals is 50000 counts and $\alpha=0.05$.

Fig. 9 shows an example of the unfolding result of the pulse height spectrum for ${ }^{22} \mathrm{Na}$ with the sequential Bayesian estimation in 50000 counts, $\alpha=0.05$. In the figure, two peaks of $0.511 \mathrm{MeV}$ and $1.27 \mathrm{MeV}$ are observed, which correspond to the two emitted gammaray energies for ${ }^{22} \mathrm{Na}$. The obtained ratio of the number of emitted gamma-rays for the two energies is 0.511 $\mathrm{MeV}: 1.27 \mathrm{MeV}=1.99: 1$. Though the ratio is a little larger than the literature value, the agreement is acceptable. Also, it was found that the result of the sequential Bayesian estimation fluctuates depending on the number of counts, that is, the estimation is a little unstable for the detector count. We should carefully examine this point in the next step. However, from the examination carried out so far in such as the result of Fig. 9 , it was confirmed that the sequential Bayesian estimation would have a potential ability to apply to realtime and simultaneous gamma-ray dose and spectrum estimation.

\section{Conclusions}

We have been developing a new spectrometer which can display the energy spectrum and dose of gamma-rays at the same time in real time. As a detection device of gamma-rays, $\mathrm{CsI}(\mathrm{Tl})$ scintillator $\left(3 \times 3 \times 3 \mathrm{~cm}^{3}\right)$ was chosen because the basic performance of $\mathrm{CsI}(\mathrm{Tl})$, i.e., the detection efficiency and energy resolution, was similar to commonly used $\mathrm{NaI}(\mathrm{Tl})$ scintillator. The $\mathrm{CsI}(\mathrm{Tl})$ crystal was attached to a MPPC in an aluminum box to constitute a prototype detector. By using this detector, measurements were carried out to test the basic performance. After that, the possibility of data processing in real time was examined by application of the Bayesian estimation. As the result, we found that the sequential Bayesian estimation could realize measurement of gamma-ray energy spectrum and dose in real time. As a future work, we will carry out a more detailed measurement and thereafter, develop an actual spectrometer.

\section{References}

1. T. Nakamori, et al. Development of a gamma-ray imager using a large area monolithic $4 \times 4$ MPPC array for a future PET scanner, JINST 7 C01083(2012)

2. S. Iwasaki, A new approach for unfolding problems based only on the Bayes' Theorem, Proceedings of the $9^{\text {th }}$ International Symposium on Reactor Dosimetry, pp. 245-252 (1996)

3. X-5 Monte Carlo Team, MCNP - A General NParticle Transport Code, Version 5, Los Alamos National Laboratory, LA-UR-03-1987 (2003)

4. R.J.D.Beattie, J.Byrne., A Monte Carlo program for evaluating the response of a scintillation counter to monoenergetic gamma rays, Nuclear Instrument and Method 104, 163-168, (1972) 